ORIGINAL ARTICLE

\title{
Anti-basal ganglia antibodies: a possible diagnostic utility in idiopathic movement disorders?
}

\author{
A J Church, R C Dale, G Giovannoni
}

Arch Dis Child 2004;89:611-614. doi: 10.1136/adc.2003.031880

See end of article for authors' affiliations .....................

Correspondence to: Mr A Church,

Neuroimmunology Laboratory, Institute of Neurology, London WCIN 3BG, UK: A.Church@ion.ucl.ac.uk

Accepted 27 September 2003

\begin{abstract}
Background: The spectrum of post-streptococcal brain disorders includes chorea, tics, and dystonia. The proposed mediators of disease are anti-basal ganglia (neuronal) antibodies (ABGA).

Aim: To evaluate ABGA as a potential diagnostic marker in a cohort of UK post-streptococcal movement disorders.

Methods: Forty UK children presenting with movement disorders associated with streptococcal infection were recruited. ABGA was measured using ELISA and Western immunoblotting. To determine ABGA specificity and sensitivity, children with neurological diseases $(n=100)$, children with uncomplicated streptococcal infection $(n=40)$, and children with autoimmune disease $(n=50)$ were enrolled as controls. Results: The mean ELISA result was increased in the post-streptococcal movement disorder group compared to all controls and derived a sensitivity of $82.4 \%$ and specificity of $79 \%$. The Western immunoblotting method to detect ABGA derived a sensitivity and specificity of $92.5 \%$ and $94.7 \%$ respectively. There was common binding to basal ganglia antigens of 40,45 , and $60 \mathrm{kDa}$. Immunofluorescence localised the antibody binding to basal ganglia neurones.

Conclusion: ABGA appears to be a potentially useful diagnostic marker in post-streptococcal neurological disorders. Western immunoblotting appears to be the preferred method due to good sensitivity and specificity and the ability to test several samples at once.
\end{abstract}

$\mathrm{S}$ ydenham's chorea (SC) is the classical post-streptococcal neurological disorder occurring weeks to months after group A $\beta$ haemolytic streptococcal (GABHS) infection and is one of the major diagnostic criteria of rheumatic fever. In addition to chorea, neuropsychiatric manifestations are common, particularly obsessive-compulsive disorder (OCD). ${ }^{1}$ A new post-streptococcal brain syndrome has recently been proposed and termed: paediatric autoimmune neuropsychiatric disorders associated with streptococcal infections (PANDAS). Patients with PANDAS are characterised by the acute onset of tics and co-morbid neuropsychiatric symptoms, particularly OCD and a relation to streptococcal infections. SC and PANDAS are both thought to be precipitated by GABHS, a common infectious agent in childhood. ${ }^{1}$

The proposed mechanism of post-streptococcal CNS disorders is that antibodies are produced against streptococcal epitope(s) which bind to basal ganglia antigen(s) due to antigenic similarity. ${ }^{2}$ It has been proposed that the antibody causes either basal ganglia dysfunction or destruction through a process of molecular mimicry driven autoimmunity. ${ }^{2}$ This hypothesis is supported by the presence of serum IgG from patients with SC, PANDAS, and post-streptococcal dystonia which reacts specifically against basal ganglia neurones with little reactivity in controls. ${ }^{35-7}$

Consistent with previous studies, we have recently shown a high prevalence of anti-basal ganglia antibodies (ABGA) using a sensitive and specific Western immunoblotting method in a cohort of Brazilian patients with SC. ${ }^{7}$ Furthermore, we have proposed that only a restricted number of basal ganglia antigens are involved in antibody binding, rather than a polyspecific response. ${ }^{7}$ We believe that ABGA may have potential as a diagnostic marker in identifying patients with post-streptococcal movement disorders and may be responsible for a broad spectrum of dyskinetic phenotypes. ${ }^{58}$ Whether ABGA are central to the pathology of these disorders or serve as a surrogate marker for atypical dyskinesias is still unknown.

We evaluated the presence of ABGA by ELISA, Western immunoblot, and standard immunofluorescence methods in a sequential UK cohort of children presenting with clinically defined post-streptococcal chorea or tics. Extensive control groups were also investigated and ABGA results from all groups were analysed to determine the diagnostic role (sensitivity and specificity) of using ABGA routinely in the investigation of "idiopathic" childhood movement disorders.

\section{METHODS}

\section{Patients and controls}

Forty children were examined by RCD between 1999 and 2002 and had a diagnosis of either chorea $(n=20)$, tics $(n=$ 16), or dystonia/tremor/stereotypies/opsoclonus-myoclonus $(\mathrm{n}=1$ each). The movement disorders occurred shortly after GABHS throat infection. The post-streptococcal patients were defined as those who had a new onset movement disorder following streptococcal infection. Streptococcal infection was defined by the presence of clinical signs (pharyngitis), throat culture, and/or raised streptococcal serology. All patients had clinical signs of GABHS infection; positive throat culture for GABHS was found in six patients and raised streptococcal serology in 40 (increased ASOT or DNase B with reduction on convalescence in all cases). All the patients with tics fulfilled the criteria for PANDAS; ${ }^{1}$ those with chorea were diagnosed with SC and the remaining patients had "idiopathic" movement disorders.

Abbreviations: $A B G A$, anti-basal ganglia antibodies; $G A B H S$, group $A$ $\beta$ haemolytic streptococcus; $O C D$, obsessive-compulsive disorder; PANDAS, paediatric autoimmune neuropsychiatric disorders associated with streptococcal infections; SC, Sydenham's chorea; TS, Tourette's syndrome 
During the same time period 100 neurological disease patients were recruited as controls (table 2). In addition children with uncomplicated streptococcal infection $(\mathrm{n}=40)$ and children with autoimmune disease $(n=50)$ were also enrolled (tables 2 and 3). Patient and controls were from the same tertiary referral centre GOSH and all patients and controls signed consent for this study, which was authorised by the local ethics committee. Whole blood samples were taken and stored at $-20^{\circ} \mathrm{C}$ in the same manner until used. These control groups have been published previously. ${ }^{18}$

All patients were tested to exclude other causes of their movement disorder. This involved excluding other autoimmune conditions such as SLE by routinely testing for antinuclear and other systemic autoantibodies. Family and personal histories were also carefully investigated by RCD to ensure hereditary movement disorders were not present. The clinical phenotypes are presented in detail elsewhere. ${ }^{20}$ The demographics of the post-streptococcal dyskinesia patients are presented in table 1 and the control groups are summarised in tables 2 and 3.

\section{Anti-basal ganglia antibodies}

The ABGA, ELISA, Western immunoblotting, and indirect immunofluorescence methods have been previously described. $^{57}$ The antigen used in all methods was derived from human basal ganglia (caudate and putamen) and came from a donor with no history or evidence of neurological disease.

We have previously ascertained that basal ganglia homogenates from different donors give the same results when using a protein concentration of $30 \mu \mathrm{g}$ per gel in Western immunoblotting and $1 \mu \mathrm{g} / \mathrm{ml}$ in ELISA (data not shown). ${ }^{57}$ Serum samples were diluted 1/300 for Western blotting and ELISA in all patients and controls. For indirect immunofluorescence, serum was diluted $1 / 25$ in all patients and controls. The patient and control samples were all coded and testing was performed with clinical data blinded.

To ensure testing against basal ganglia neuronal proteins in ELISA and Western immunoblotting the homogenate was mixed with di-iso-propyl-ether (VWR, UK) and centrifuged at $3000 \mathrm{rpm}$ for 10 minutes to remove the lipid fraction from the supernatant. The protein fraction was collected and stored at $-80^{\circ} \mathrm{C}$ until required. Sections of human basal ganglia were cut from a frozen block containing caudate and putamen; $10 \mu \mathrm{m}$ sections were prepared from the sample block and stored at $-80^{\circ} \mathrm{C}$ until use. A known SC positive and normal control were tested on each method to act as quality controls.

\section{Statistics}

All statistics were performed using SPSS. ABGA, ELISA results were analysed using the non-parametric two sample exact Wilcoxon rank-sum test. ABGA Western immunoblotting results from patients and controls were compared using the $\chi^{2}$ test.

\begin{tabular}{|c|c|c|c|}
\hline Group & Number & $\operatorname{Sex}(M / F)$ & $\begin{array}{l}\text { Mean age, years } \\
\text { (range) }\end{array}$ \\
\hline $\begin{array}{l}\text { Tics } \\
\text { Chorea } \\
\text { Idiopathic movement } \\
\text { disorders }\end{array}$ & $\begin{array}{r}16 \\
20 \\
4\end{array}$ & $\begin{array}{l}11 / 5 \\
7 / 13 \\
3 / 1\end{array}$ & $\begin{array}{l}6.75(2-13) \\
7.85(1.3-14) \\
7.1(1.2-16)\end{array}$ \\
\hline
\end{tabular}

\section{RESULTS}

\section{ABGA ELISA}

ELISA provides a semi-quantitative measure of antibody reactivity against a prepared homogenate of whole human basal ganglia. The ELISA mean absorbance results in patients and controls are presented in table 4.

The mean ABGA ELISA result was increased in the poststreptococcal movement disorder group compared to the neurological, streptococcal, and autoimmune controls $(\mathrm{p}<0.0001$ in all comparisons $)$. In addition analysis of the post-streptococcal group showed that the mean ABGA ELISA was increased in those patients tested during acute disease (mean absorbance $0.445,95 \%$ CI 0.373 to 0.517 ), compared to patients who developed a persistent disease (mean absorbance $0.294,95 \%$ CI 0.255 to 0.333 ) ( $p<0.005$ ). This is in accordance with the reduction in positivity reported in persistent compared to acute SC. ${ }^{7}$ The ABGA ELISA derived a sensitivity of $82.4 \%$ and specificity of $79 \%$.

\section{ABGA Western immunoblotting (table 4)}

Reactivity against any basal ganglia antigen was more prevalent in the post-streptococcal dyskinesia patients compared to controls ( $p<0.0005)$. ABGA was positive in 94\% (15/16) of tics (PANDAS) and 95\% (19/20) of chorea (Sydenham's chorea) compared to 5\% (8/150) of all the control groups combined. Western immunoblotting showed antibody reactivity to specific basal ganglia antigens in the movement disorder patients rather than a polyspecific response. This method derived a sensitivity of $93 \%$ and specificity of $94.7 \%$ in detecting post-streptococcal movement disorders.

There were three common basal ganglia antigens detected in the patients $(40,45,60 \mathrm{kDa})$. There was no difference in the antigen binding pattern between tic or chorea phenotypes using the $45 \mathrm{kDa}$ and $60 \mathrm{kDa}$ antigens. In contrast, reactivity to the $40 \mathrm{kDa}$ antigen did appear to be more predominant in the patients with tics rather than chorea as reactivity to this antigen was present in $62.5 \%(10 / 16)$ compared to $30 \%(6 / 20)$ of the chorea patients $(\mathrm{p}<0.01)$.

Four per cent $(4 / 100)$ of the neurological controls were ABGA Western immunoblotting positive. The clinical presentations were: status dystonicus $(n=1)$, encephalitis $(\mathrm{n}=1)$, ataxia $(\mathrm{n}=1)$, and leukodystrophy $(\mathrm{n}=1)$. Only the patient with encephalitis had binding to the 40 and $60 \mathrm{kDa}$ basal ganglia antigens, while the other patients bound to a number of antigens (table 4$)$. Two per cent $(1 / 40)$ of the streptococcal controls were also positive and bound to 35 and $60 \mathrm{kDa}$ antigens. Ten per cent (5/50) of the autoimmune controls were also positive. Two patients had rheumatic fever and had reactivity to 40,50 , and $60 \mathrm{kDa}$ basal ganglia antigens, two patients had glomerulonephritis and binding to 35 and $40 \mathrm{kDa}$ antigens, and one patient had arthritis and binding to a $40 \mathrm{kDa}$ antigen (table 4). All the positive patients from the autoimmune group had post-streptococcal diseases.

\section{CSF Western immunoblotting}

The patient with an "idiopathic" movement disorder (tics and stereotypies) who had a negative serum ABGA Western immunoblot had CSF available for examination. This showed positive IgG binding against a $40 \mathrm{kDa}$ basal ganglia antigen. CSF was not otherwise routinely examined.

\section{Diagnostic utility of ABGA in movement disorders}

As Western immunoblotting had high sensitivity and specificity in post-streptococcal movement disorders, we evaluated this method as a diagnostic tool in children with new onset, otherwise "idiopathic" movement disorders. Those patients with a clinical diagnosis of post-streptococcal 
Table 2 Neurology controls, clinical diagnosis $(n=100)$

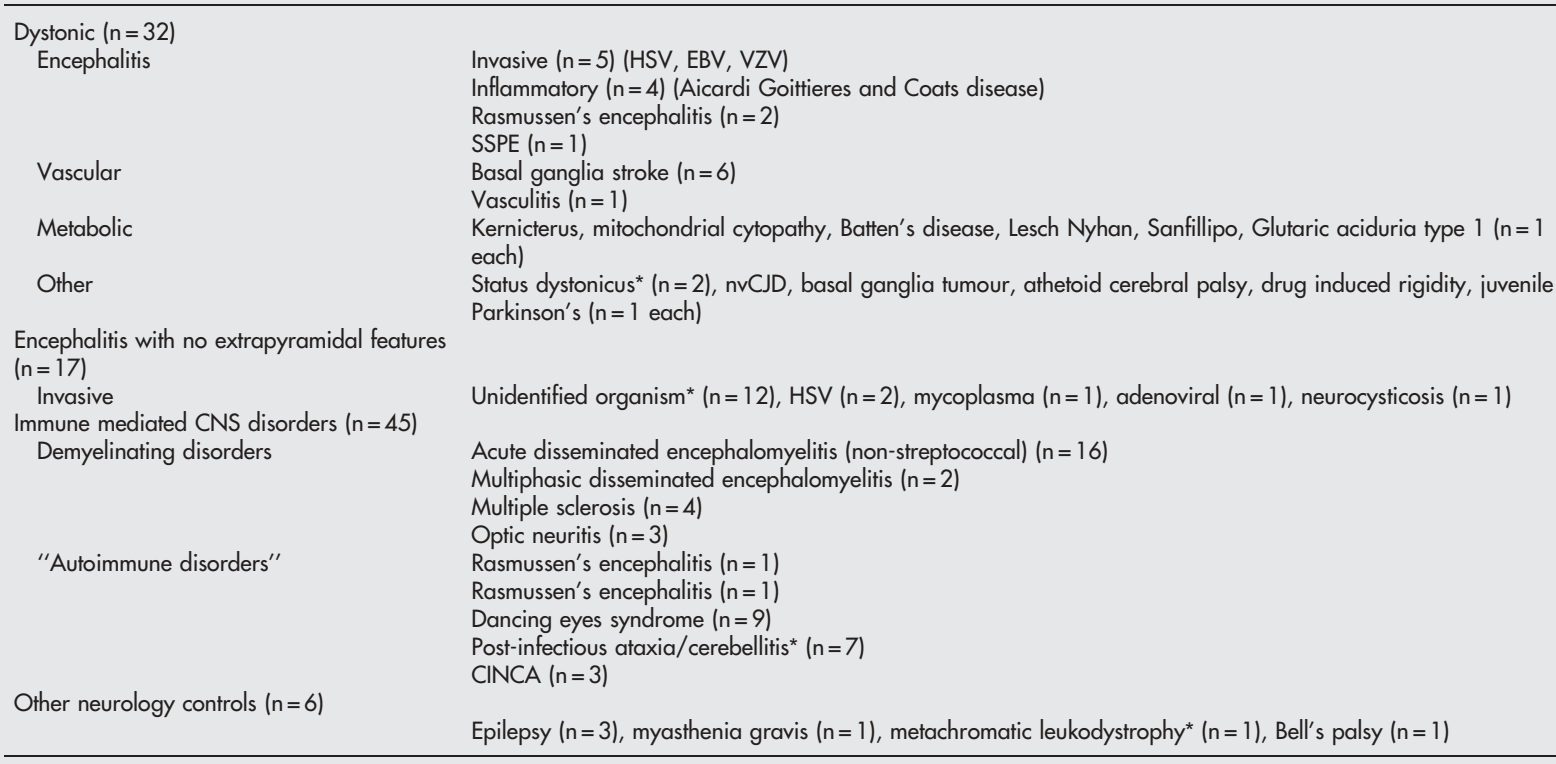

Patients denoted with * had positive ABGA Western immunoblotting.

movement disorders $(n=40)$ were compared with the dystonic, neurology control group $(n=32)$. The diagnostic sensitivity of ABGA was $93 \%$ and specificity $97 \%$. The positive predictive value was $97 \%$ (likelihood ratio for a positive test $=31)$, and the negative predictive value was $91 \%$ (likelihood ratio for a negative test $=0.072$ ).

\section{ABGA immunofluorescence}

Indirect immunofluorescence was performed in five patients with chorea and five with tics. The same binding pattern was seen in all patients tested with IgG binding to large caudate head neurones, with less predominant binding to neurones in the putamen. The binding pattern appeared to be axonal, with sparing of the cell body and predominant cytoplasmic staining.

\section{DISCUSSION}

Antibodies reactive against basal ganglia were originally reported using frozen sections of human basal ganglia (caudate and subthalamic nucleus) in patients with acute SC. ${ }^{3}$ Two further studies using human basal ganglia have subsequently showed that ABGA are probably universal in acute $\mathrm{SC} ;{ }^{7}$ although antibodies appeared to be less prevalent in patients with persistent chorea. ${ }^{67}$ We have previously shown in a Brazilian cohort of SC that the auto-antigens appear to be specific to (or enriched) in the basal ganglia compared to other brain regions. ${ }^{7}$ Furthermore, three dominant basal ganglia antigens of molecular weights 40 , 45 , and $60 \mathrm{kDa}$ appear to be involved in antibody-antigen binding as opposed to polyspecific binding. ${ }^{7}$

In this study we sought to investigate whether ABGA could be detected and used diagnostically in a UK cross-sectional study of post-streptococcal chorea and tics (PANDAS). We found a similar immunofluorescence binding pattern against basal ganglia neurones to that previously reported in SC. ${ }^{367}$ This may suggest that regardless of the movement disorder phenotype (Sydenham's chorea or PANDAS), the associated ABGA response appeared to be essentially the same. It is not surprising that antibody binding could produce a range of extra-pyramidal movements, as diseases affecting the basal ganglia rarely result in just one type of movement disorder phenotype. 9
ABGA ELISA allowed for several samples to tested at once, but sensitivity and specificity were considerably less than IF and Western immunoblotting. Antibody binding to non-basal ganglia specific proteins can also not be ruled out. In this study there were low levels of antibody reactivity using ELISA in control groups. This may reflect low levels of ABGA in normal controls or lack of assay sensitivity. Analysis of the Western blotting results indicated that the same antigens were present in either tics or chorea, although the $40 \mathrm{kDa}$ basal ganglia antigen was more common in patients with post-streptococcal tics (PANDAS). The presence of distinct antigens in PANDAS or SC patients could support a pathology effecting different basal circuits. Alternatively the three antigens we have recognised in this study and previously $^{7}$ may just represent different epitopes of the same protein or SDS produced fractions. Identifying the 40,45 , and $60 \mathrm{kDa}$ proteins is necessary to improve our understanding of these syndromes, and help establish whether ABGA has functional or destructive effects on neurones.

The presence of ABGA has also been shown somewhat controversially in Tourette's syndrome (TS), which is an important concept given the clinical similarity of PANDAS to TS. ${ }^{10-14}$ These results have not always been reproduced, which may cast doubt as to the pathogenic role of ABGA, or could reflect the differences in current methodology used to detect anti-neuronal antibodies. ${ }^{13}{ }^{14}{ }^{18}$ We have also found that $25 \%$

Table 3 Autoimmune controls without neurological complications $(n=50)$

\begin{tabular}{ll}
\hline Clinical phenotype & Number \\
\hline Rheumatic fever, no chorea* & $\mathrm{n}=16$ \\
Post-streptococcal glomerulonephritis* & $\mathrm{n}=10$ \\
Post-streptococcal vasculitis/erythema nodosum & $\mathrm{n}=5$ \\
Post-streptococcal arthritis* & $\mathrm{n}=4$ \\
Post-streptococcal uveitis & $\mathrm{n}=1$ \\
Juvenile idiopathic arthritis & $\mathrm{n}=4$ \\
Henoch-Schonlein purpura & $\mathrm{n}=4$ \\
Systemic lupus erythematosus & $\mathrm{n}=3$ \\
Kawasaki disease, polyarteritis nodosa, Cogan's & $\mathrm{n}=1$ each \\
syndrome & \\
\hline Patients denoted with * had positive ABGA Western immunoblotting.
\end{tabular}


Table 4 Streptococcal and anti-basal ganglia antibody results

\begin{tabular}{|c|c|c|c|c|}
\hline Group & $\begin{array}{l}\text { Post-streptococcal } \\
\text { autoimmune dyskinesia } \\
(\mathrm{n}=40)\end{array}$ & Neurology controls $(n=100)$ & $\begin{array}{l}\text { Uncomplicated streptococcal } \\
\text { infection }(n=40)\end{array}$ & Autoimmune controls $(n=50)$ \\
\hline \multicolumn{5}{|l|}{ ABGA ELISA } \\
\hline Mean $(95 \% \mathrm{Cl})$ & $0.396(0.341$ to 0.451$)$ & $0.229(0.207$ to 0.251$)$ & $0.189(0.171$ to 0.207$)$ & 0.251 (0.20 to 0.302$)$ \\
\hline \multicolumn{5}{|l|}{ ABGA Western B } \\
\hline$\%$ positive & $92.5 \%$ & $4 \%$ & $2 \%$ & $10 \%$ \\
\hline \multicolumn{5}{|l|}{ Antigen binding } \\
\hline $40 \mathrm{kDa}$ & $47.5 \%$ & $1 \%$ & $0 \%$ & $4 \%$ \\
\hline $45 \mathrm{kDa}$ & $40 \%$ & $0 \%$ & $0 \%$ & $0 \%$ \\
\hline $60 \mathrm{kDa}$ & $42.5 \%$ & $1 \%$ & $2 \%$ & $2 \%$ \\
\hline $80 \mathrm{kDa}$ & $25 \%$ & $0 \%$ & $0 \%$ & $0 \%$ \\
\hline Other & $30,35,42,62,95 \mathrm{kDa}$ & $42,43,95 \mathrm{kDa}$ & $35 \mathrm{kDa}$ & $35,50 \mathrm{kDa}$ \\
\hline
\end{tabular}

of patients with TS bind to 40, 45, and $60 \mathrm{kDa}$ human basal ganglia antigens and have raised streptococcal serology which may suggest that a subset of TS patients has a poststreptococcal autoimmune pathology. ${ }^{18}$ The concept of an autoimmune pathology in TS is one which deserves attention as it may lead to alternative therapies for TS at least in some subtypes of the syndrome.

In this UK cohort of defined post-streptococcal dyskinesias, ABGA appear to be prevalent during the active phase of disease, and decrease during remissions (data not shown). This phenomenon may be important when attempting to identify autoimmune subgroups in TS or OCD. As Western immunoblotting can define specific antigenic binding, we have found this tool, in conjunction with immunofluorescence to be our preferred diagnostic marker. The prevalence of ABGA using these methods in 150 controls studied was low $(5 \%)$.We believe that Western immunoblotting and immunofluorescence is preferred to the ELISA, which cannot define specific antigenic binding.

Showing the presence of ABGA does not of course infer pathogenicity as the antibody may be produced as a consequence of local damage. Potential, functional effects have been suggested by two studies describing stereotypical movements in rats after infusion of IgG from patients with PANDAS. ${ }^{15}{ }^{16}$ Furthermore, the potential removal of circulating IgG using plasmapheresis and intravenous immunoglobulin has been reported to improve symptoms in PANDAS patients. ${ }^{17}$ A recent report has also suggested monoclonal antibodies raised from a patient with SC reacted to the neuronal antigen lysoganglioside and $\mathrm{N}$-acetyl- $\beta$-D-glucosamine, which is found on the GABHS cell surface. The implications of this finding in PANDAS and SC needs to be investigated further. ${ }^{19}$ These important studies suggest that the antibodies could be pathogenic. The majority of investigations to date have focused on an antibody hypothesis, although alternative mechanisms of action including superantigen mediated disease or cell mediated immunity have yet to be explored in depth.

In conclusion, this study may suggest that ABGA Western immunoblotting could be used as a surrogate marker in diagnosing patients with idiopathic movement disorders where streptococcal infection has either been implicated as an initial trigger or associated with exacerbations. This may suggest that cases of dyskinesia where other causes have been eliminated and where there is strong suspicion of a streptococcal trigger should be investigated as to whether they fit the PANDAS hypothesis. Importantly the range of dyskinetic outcomes should not be limited to just that of chorea or tics.

\section{Authors' affiliations}

A J Church, R C Dale, G Giovannoni, Neuroinflammation Department, Institute of Neurology, Queen Square, London, UK WCIN 3BG

\section{REFERENCES}

1 Swedo SE, Leonard HL, Garvey M, et al. Paediatric autoimmune neuropsychiatric disorders associated with streptococcal infections: clinical description of the first 50 cases. Am J Psychiatry 1998;155:264-71.

2 Bronze MS, Dale JB. Epitopes of streptococcal M proteins that evoke antibodies that cross-react with human brain. J Immunol 1993;151:2820-8.

3 Husby G, van de Rijn I, Zabriskie JB, et al. Antibodies reacting with cytoplasm of subthalamic and caudate nuclei neurons in chorea and acute rheumatic fever. J Exp Med 1976;144:1094-110.

4 Kiessling LS, Marcotte AC, Culpepper L. Antineuronal antibodies in movement disorders. Pediatrics 1993;92:39-43.

5 Dale RC, Church AJ, Cardoso F, et al. Poststreptococcal acute disseminated encephalomyelitis with basal ganglia involvement and auto-reactive antibasal ganglia antibodies. Ann Neurol 2001;50:588-95.

6 Kotby AA, El Badawy N, El Sokkary S, et al. Antineuronal antibodies in rheumatic chorea. Clin Diagn Lab Immunol 1998;5:836-9.

7 Church AJ, Cardoso F, Dale RC, et al. Anti-basal ganglia antibodies in acute and persistent Sydenham's chorea. Neurology 2002;59:227-31.

8 Dale RC, Church AJ, Benton S, et al. Post-streptococcal autoimmune dystonia with isolated bilateral striatal necrosis. Dev Med Child Neurol 2002;44:485-9.

9 van Dijk JG, van der Velde EA, Roos RA, et al. Juvenile Huntington disease. Hum Genet 1986;73:235-9.

10 Singer HS, Giuliano JD, Hansen BH, et al. Antibodies against human putamen in children with Tourette syndrome. Neurology 1998;50:1618-24.

11 Wendlandt JT, Grus FH, Hansen BH, et al. Striatal antibodies in children with Tourette's syndrome: multivariate discriminant analysis of $\lg G$ repertoires. J Neuroimmunol 2001;119:106-13.

12 Morshed SA, Parveen S, Leckman JF, et al. Antibodies against neural, nuclear, cytoskeletal, and streptococcal epitopes in children and adults with Tourette's syndrome, Sydenham's chorea and autoimmune disorders. Biol Psychiatry 2001;50:566-77.

13 Scahill L, Lombroso PJ. Antibodies against neural, nuclear, cytoskeletal, and streptococcal epitopes in children and adults with Tourette's syndrome, Sydenham's chorea, and autoimmune disorders. Biol Psychiatry 2001;50:566-77.

14 Singer HS, Giuliano JD, Hansen BH, et al. Antibodies against a neuron-like (HTB-10 neuroblastoma) cell in children with Tourette syndrome. Biol Psychiatry 1999;46:775-80.

15 Hallett JJ, Harling-Berg CJ, Knopf PM, et al. Anti-striatal antibodies in Tourette syndrome cause neuronal dysfunction. J Neuroimmunol 2000;111:195-202.

16 Taylor JR, Morshed SA, Parveen S, et al. An animal model of Tourette's syndrome. Am J Psychiatry 2002;159:657-60.

17 Perlmutter SJ, Leitman SF, Garvey MA, et al. Therapeutic plasma exchange and intravenous immunoglobulin for obsessive-compulsive disorder and tic disorders in childhood. Lancet 1999;354:1153-8.

18 Church AJ, Dale RC, Lees AJ, et al. Tourette's syndrome: a cross sectional study to examine the PANDAS hypothesis. I Neurol Neurosurg Psychiatry 2003;74:602-7.

19 Kirvan CA, Swedo SE, Heuser JS, et al. Mimicry and autoantibodymediated neuronal cell signalling in Sydenham chorea. Nature Med 2003;9:914-20.

20 Dale RC, Heyman I, Surtees RAH, et al. Dyskinesias and associated psychiatric disorders following streptococcal infections. Arch Dis Child 2004;89:604-10. 